\title{
Care of Child with Hypoxic Ischemic Encephalopathy
}

\section{Shweta}

M.Sc Tutor, St. Stephen's Hospital College of Nursing, Tis Hazari, Delhi, India

Keywords: Hypoxic Ischemic Encephalopathy, Mental Retardation, Cerebral Palsy, Epilepsy, Morbidity

\section{Corresponding author}

\section{Shweta}

M.Sc Tutor, St. Stephen's Hospital College of Nursing, Tis Hazari, Delhi, India

Email: shweta.gahalian15@gmail.com

\begin{abstract}
Qypoxic Ischemic Encephalopathy (HIE) is a type of brain damage that - 0 occurs when an infant's brain doesn't receive enough oxygen and blood. It is a dangerous condition that requires immediate medical intervention. Neonatal encephalopathy affects 2 to 5 of every 1000 live births and represents a major cause of mortality and long-term morbidity in affected infants. Hypoxic ischemic encephalopathy (HIE) is the major cause of encephalopathy in the neonatal period. Until recently, management of a newborn with encephalopathy has consisted largely of supportive care to restore and maintain cerebral perfusion, provide adequate gas exchange and treat seizure activity. Twenty five percent of neonates with HIE develop severe and permanent neuropsychological sequelae, including mental retardation, cerebral palsy, and epilepsy.

Hypoxic-ischemic encephalopathy causes significant morbidity and mortality in neonates. Preventing the secondary reperfusion injury that occurs following a hypoxic-ischemic event is paramount to ensuring the best possible neurologic outcome for the neonate. Induced hypothermia is currently being studied in various institutions as a means of neuroprotection for neonates at risk of severe brain injury following a hypoxic-ischemic event. This article highlights the pathophysiology of hypoxic-ischemic encephalopathy and the rationale behind the effectiveness of induced hypothermia. Nursing care and management of neonates being treated with induced hypothermia are discussed (Long and Brandon, 2007).
\end{abstract}

Perinatal hypoxic-ischemic encephalopathy (HIE) is a syndrome of disturbed neurological function in the early life characterized by clinical and laboratory proof of acute or subacute brain injury (Vries and Jongmans, 2010).

Despite major advances in monitoring technology and knowledge of fetal and neonatal pathologies, hypoxicischemic encephalopathy (HIE) remains a serious condition that causes significant mortality and long-term morbidity (Ellis et al. 2000).

Hypoxic Ischemic Encephalopathy (HIE) occurs at a rate of approx.1-2 /1000 full term live birth in high income countries in low income countries the incidence is much higher. HIE is of concern because it can lead to serious long-term neuro-motor sequelae among survivors.

Neonatal hypoxic ischemic encephalopathy (HIE) is a devastating disease that primarily causes neuronal and white matter injury. HIE has tremendous detrimental effects on the developing brain and is among the leading causes of death among infants, as well as the major underlying cause of seizures in term infants (Shetty, 2015).

Neonatal HIE can also be characterized as an injury that occurs in the immature brain, resulting in delayed cell death via excitotoxicity, inflammation, and oxidative stress (Northington et al. 2011). 


\section{Definition}

HIE is a brain dysfunction caused by a reduction in the supply of oxygen to the brain and other organs (hypoxia), compounded by low blood flow to vital organs (ischemia). Encephalopathy refers to any condition that results from reduced blood and oxygen supply to the brain.

\section{Incidence}

Overall, the relative incidence of neonatal encephalopathy is estimated to be between 2 and 9 per 1000 term births (www.uptodate.com). $40 \%$ to $60 \%$ of affected infants die by 2 years old or have severe disabilities (Allen et al. 2011). In 2013, it was estimated to have resulted in 644,000 deaths down from 874,000 deaths in 1990.

\section{Pathophysiology}

Brain hypoxia and ischemia due to systemic hypoxemia, reduced cerebral blood flow $(\mathrm{CBF})$, or both are the primary physiologic processes that lead to hypoxic-ischemic encephalopathy (HIE) (Ferriero and Perlman, 2004).

The initial compensatory adjustment to an asphyxial event is an increase in CBF due to hypoxia and hypercapnia. This is accompanied by a redistribution of cardiac output to essential organs, including the brain, heart, and adrenal glands. A blood pressure (BP) increase due to increased release of epinephrine further enhances this compensatory response. See the image below.

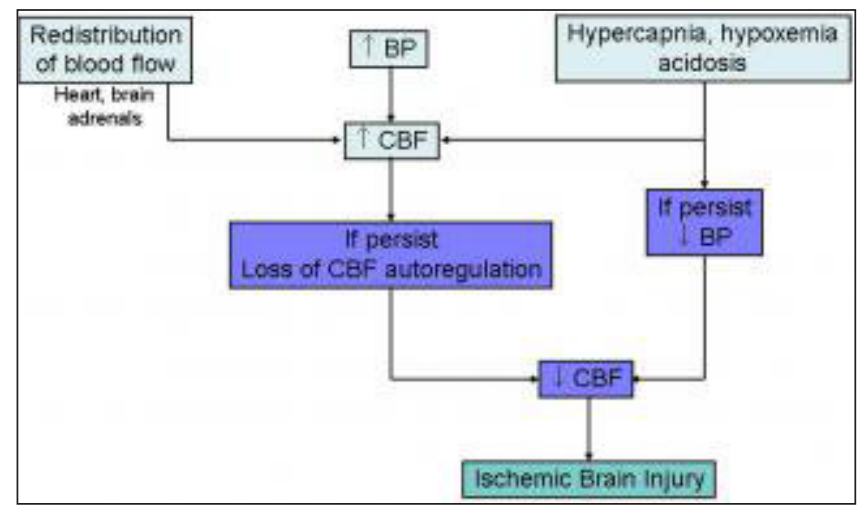

\section{Clinical Features}

There are a number of symptoms associated with HIE, including:

" Meconium-stained amniotic fluid $\rightarrow$ Low heart rate

Poor muscle tone

W Weak breathing or no breathing at all

- Bluish or pale skin color

- Excessive acid in the blood

At delivery, the presence of yellow, meconium stained amniotic fluid is evidence that fetal distress has occurred. At birth, these infants are frequently depressed and fail to breathe spontaneously. During the ensuring hours, they may remain hypotonic or change from hypotonic to hypertonic, or their tone may appear normal (Kliegman et al.).

\section{Causes}

Various problems or medical complications may cause HIE during pregnancy in the antepartum period. These include:

- Maternal diabetes with vascular disease

" Problems with blood circulation to the placenta

" Preeclampsia

* Cardiac disease

" Congenital infections of the fetus

D Drug and alcohol abuse

" Severe fetal anemia

" Lung malformations

HIE can also affect infants during labor and delivery, or what is called the intrapartum period. Common intrapartum complications may include:

मExcessive bleeding from the placenta

- Very low maternal blood pressure

- Umbilical cord accidents

- Prolonged late stages of labor

- Abnormal fetal position

D Rupture of the placenta or the uterus

In addition, Infants can suffer from the effects of HIE in the postpartum period. The most common causes of postpartum HIE include:

- Severe cardiac or pulmonary disease

"Infections, including sepsis and meningitis

" Severe prematurity

\Low neonatal blood pressure

$\rightarrow$ Brain or skull trauma 
Table 1: Sarnart Scoring System (Kliegman et al.)

\begin{tabular}{cccc}
\hline & Hypoxic Ischemic Encephalopathy in Term Infants Sarnart Scoring System & STAGE 3 \\
\hline SIGNS & STAGE 1 & STAGE 2 & Coma \\
Level of consciousness & Hyperalert & Lethargic & Flaccid \\
Muscle tone & Normal & Hypotonic & Decerebrate \\
Posture & Normal & Flexion & Absent \\
Tendon reflex/ clonus & Hyperactive & Hyperactive & Absent \\
Myoclonus & Present & Present & Absent \\
Moro reflex & Strong & Weak & Unequal, poor light reflex \\
Pupils & Mydriasis & Miosis & Decerebration \\
Seizures & None & Common & Burst suppression to isoelectric \\
Electroencephalographic & Normal & Low voltage changing to seizure & activity \\
Duration & $<24$ hours if progresses; & 24 hours to 14 days & Days to weeks
\end{tabular}

Congenital brain malformations (Birth Injury Guide HIE).

\section{Diagnosis}

Tests and studies are conducted once medical professionals have observed signs and symptoms of HIE. These include:

- CT scan: This test uses x-rays and computer software to make pictures of the baby's brain.

- MRI scan: This test uses magnetic waves to make pictures of structures inside the baby's brain.

- EKG (electrocardiogram): This test records heart activity by measuring electrical currents through the heart muscle.

- Blood tests (arterial blood gases): These tests are used to help determine how well the baby is breathing, the $\mathrm{pH}$ of the blood (acidic, basic, or normal), and if any acidemia present is due to breathing or kidney problems.

- Blood glucose levels: Excessively high glucose levels can lead to brain injury.

- EEG (electroencephalogram): This test records the activity of the baby's brain by measuring electrical currents through the brain.

- Ultrasound: This test uses sound waves to evaluate blood flow in the vessels going to the brain or within the brain.

W Evoked potential test: This is a neurophysiological test that evaluates the functioning of the baby's brain by recording brain waves.

D Echocardiogram: This test uses high-frequency sound waves to examine the size, shape, and motion of the baby's heart (//www.abclawcenters.com/ frequently-asked-questions/how-is-hie).

\section{Management}

Initial management includes effective resuscitation at the delivery room followed by supportive care under frequent monitoring.

Temperature: even through hypothermia has been recently investigated for its neuroprotective actions presently recommended temperature remains the thermoneutral zone for the baby (Kliegman et al.).

Carbon-dioxide: Hypercarbia cause cerebral acidosis and vasodilatation, which leads to stunting of blood away from compromised areas increasing infarct size. Hypocapnia of $<25 \mathrm{~mm} \mathrm{Hg}$ decreases cerebral perfusion and can cause infarction. Both should be avoided and this requires blood gas monitoring.

Perfusion: Cerebral perfusion pressure (CPP) reflects systemic blood pressure in a pressure passive manner. Hence to maintain the desired range of CPP a systemic mean arterial pressure of 45-50 mm Hg (term), 35040 (1-2 kg weight) and 30-35 mmHg (<1 kg weight) is required. Blood pressure measurement should be taken in all babies. A central venous 
pressure of 5-8 $\mathrm{mm} \mathrm{Hg}$ in term and 3-5 $\mathrm{mm} \mathrm{Hg}$ un preterm babies is ideal.

Glucose: Levels between $75-100 \mathrm{mg} / \mathrm{dl}$ are recommended. Higher level enhances cerebral edema and compromise perfusion, while hypoglycemia potentiates excitotoxic damage. Hypoglycemia is commonly seen in asphyxiated infants and must be regularly monitored.

Metabolic profile: Hypoglycemia and electrolyte disturbances should be regularly looked for till stabilization of baby and corrected as indicated.

Cerebral edema: Intracranial pressure (ICP) can be estimated by measuring the vertical distance between the anterior fontanel and the heart at the point that the midportion of the fontanel flattens when the baby is tilted up. (Normal $=50 \mathrm{~mm} \mathrm{H}_{2} \mathrm{O}$ or lower). The main cause of edema is cytotoxic injury and treatment directed at reducing the edema has not shown to be useful. However, factors enhancing it should be prevented.

Seizures: $20-50 \%$ of infants with HIE develop seizures during day 1 or 2 . Seizures are commonly focal or multifocal. Metabolic disturbances such as hypoglycemia and hyponatremia must be ruled out. Seizures may be intractable initially but usually burn themselves out by 48 hours. Major differential diagnosis includes pyridoxin deficiency and drug toxicity. Subtle seizure lasting short duration need not be treated. Once the baby is seizure free for 3-4 days anti-convulsant are stopped in the same order as they were started, except phenobarbitone. Phenobarbitone is stopped at discharge if neurological examination is normal and baby is well on breast. If it is not normal, then phenobarbitone is continue until one month (Kliegman et al).

\section{Prognosis}

The outcome of HIE correlates to the timing and severity of the insult and ranges from complete recovery to death. The prognosis varies depending on whether the metabolic and cardiopulmonary complications are treated, the infant's gestational age (outcome is poorest if the child is preterm), and the severity of the encephalopathy. A low APGAR score at $20 \mathrm{~min}$ of age, and persistence of abnormal neurologic signs at 2 weeks of age also predicts death or severe cognitive and motor deficits. Normal MRI and EEG findings are associated with a good recovery, whereas severe MRI and EEG abnormalities predict poor outcome. Infants with stage 2 and 3 encephalopathy are at the highest risk for adverse outcome. Microcephaly and poor head growth during the $1^{\text {st }}$ year of life also correlate with injury to the basal ganglia and white matter and adverse developmental outcome at 12 months. Early identification of neurodevelopmental problems allows prompt referral for developmental, rehabilitative, neurologic care, and early intervention services so that the best possible outcome can be achieved. ${ }^{10}$

\section{CONCLUSION}

HIE (Hypoxic Ischemic Encephalopathy) is caused due to lack of oxygen supply to the brain. It causes highest mortality and morbidity. It is mainly caused during pregnancy in ante partum, intra partum and post partum period. In management basically focus is on temperature recording, seizure activity, monitoring of blood glucose level and assessing cerebral edema.

\section{REFERENCES}

Long, M. and Brandon, D.H. 2007. Induced hypothermia for neonates with hypoxic-ischemic encephalopathy. Journal of Obstetric, Gynecological and Neonatal Nursing, 36(3): 293-298.

Vries, L.S. and Jongmans, M.J. 2010. Long-term outcome after neonatal hypoxic-ischaemic encephalopathy. Arch. Dis. Child Fetal Neonatal, Ed 95: F220-224.

Ellis, M., Manandhar, D.S., Wyatt, J., Bolam, A.J. and Costello, A.M. 2000. Stillbirths and neonatal encephalopathy in Kathmandu, Nepal: An estimate of the contribution of birth asphyxia to perinatal mortality in a low-income urban population. Paediatr Perinat Epidemiol., 14: 39-52.

Shetty, J. 2015. Neonatal seizures in hypoxic-ischaemic encephalopathy-risks and benefits of anticonvulsant therapy. Dev. Med. Child. Neurol., 57: 40-43.

Northington, F.J., Chavez-Valdez, R. and Martin, L.J. 2011. Neuronal cell death in neonatal hypoxia-ischemia. Ann. Neurol., 69: 743-758.

"Clinical features, diagnosis, and treatment of neonatal encephalopathy". www. uptodate.com. Retrieved 2016-03-24.

Allen, Kimberly A.; Brandon, Debra, H. 2011. "Hypoxic Ischemic Encephalopathy: Pathophysiology and Experimental Treatments". Newborn and Infant Nursing Reviews. Neuroprotective Strategies, 11(3): 125-133.

Ferriero, D.M. 2004. Neonatal brain injury. N. Engl. J. Med., 351(19): 1985-95. [Medline].

Perlman, J.M. 2004. Brain injury in the term infant. Semin Perinatol., 28(6): 415-24. 
Kliegman, Bherman, Jenson, Stantion, Nelson textbook of peadiatric, volume 1 , part $1,18^{\text {th }}$ edition, published by Elsevier, pp. 718-720.

Birth Injury Guide HIE, available on http://www. birthinjuryguide.org/birth-injury/types/hypoxicischemic-encephalopathy-hie accessed on $8^{\text {th }}$ April 2018.
Available on https://www.abclawcenters.com/frequentlyasked-questions/how-is-hie-diagnosed accessed on $8^{\text {th }}$ April 2018. 
